\title{
Lidil
}

Revue de linguistique et de didactique des langues

$64 \mid 2021$

Le passif dans la langue parlée

\section{Stratégies verbales et non verbales dans les interactions orales en intercompréhension entre langues affines}

Verbal and Non-Verbal Strategies for Oral Interactions and Intercomprehension between Sister Languages

\section{Hugues Sheeren}

\section{OpenEdition \\ Journals}

Édition électronique

URL : https://journals.openedition.org/lidil/9810

DOI : 10.4000/lidil.9810

ISSN : 1960-6052

Éditeur

UGA Éditions/Université Grenoble Alpes

Édition imprimée

ISBN : 978-2-37747-315-1

ISSN : $1146-6480$

\section{Référence électronique}

Hugues Sheeren, «Stratégies verbales et non verbales dans les interactions orales en intercompréhension entre langues affines », Lidil [En ligne], 64 | 2021, mis en ligne le 01 novembre 2021, consulté le 24 novembre 2021. URL : http://journals.openedition.org/lidil/9810 ; DOI : https:// doi.org/10.4000/lidil.9810

Ce document a été généré automatiquement le 24 novembre 2021.

(C) Lidil 


\section{Stratégies verbales et non verbales dans les interactions orales en intercompréhension entre langues affines}

Verbal and Non-Verbal Strategies for Oral Interactions and Intercomprehension between Sister Languages

Hugues Sheeren

\section{Introduction}

1 Bien que l'intercompréhension ne soit désormais plus une approche nouvelle et que de remarquables avancées dans les recherches aient été accomplies (surtout depuis une quinzaine d'années, en témoigne le nombre de publications sur le sujet), le volet oral de cette nouvelle façon d'appréhender les langues est encore peu enseigné. Plateformes, sites, méthodes, ou activités proposées en classe créées par les professeure's se concentrent majoritairement sur l'écrit (compréhension de documents/textes et sur l'interaction entre participant-es) alors que la définition même de l'intercompréhension concerne une pratique - ancestrale d'ailleurs - qui relève avant tout de l'oral.

Comme l'observe Marie-Christine Jamet, l'intercompréhension renvoie à un processus de communication, un échange oral entre des locuteurs de langues apparentées où chacun parle sa langue et comprend celle de l'autre, c'est-à-dire à une "situation de compréhension mutuelle plurilingue » (Jamet, 2009, 2010). Or, cette définition est loin de correspondre à la réalité des pratiques didactiques où prime « l'activité de lecture et pas une interaction verbale orale » ajoute-t-elle. On aboutit donc à un paradoxe puisque l'intercompréhension se définit avant tout par le fait que deux personnes puissent se comprendre et interagir oralement en parlant deux idiomes différents mais que, dans 
les faits, une bonne partie des formations, cours, ateliers consacrés à l'intercompréhension se focalisent sur la compréhension de la langue écrite. Une contradiction que ne manque pas de relever Diego Cortés Velásquez lequel, tout en signalant que certains projets axés sur l'oral existent, reconnait que «mancano sperimentazioni che forniscano dati e indicazioni circa l'efficacia e $i$ risultati delle pratiche didattiche per lo sviluppo della comprensione orale » et que "sono scarsi i materiali per l'IC orale e poche sono le ricerche effettuate » (Cortés Velásquez, 2015, p. 120).

Nombreuses sont les explications qui justifient cet état des choses ${ }^{1}$. Sandrine Caddéo, par exemple, argue que «si la dimension orale a toujours été présente, elle a, jusqu'à présent, rarement été développée avec les mêmes ambitions ", car elle implique "des paramètres plus difficilement contrôlables: système phonologique, variations de prononciation, prosodie, flux continu, débit, accent, tout un ensemble de phénomènes à partir desquels il semble moins évident d'optimiser les transparences, de favoriser les rapprochements pour accéder à une compréhension rapide, même globale " (Caddéo dans Cortés Velásquez, 2015, Prefazione, p. x). De fait, les enseignantes de langues savent qu'il existe chez les élèves un écart non seulement entre les compétences de production et de réception, mais aussi un écart entre la compréhension de l'oral et celle de l'écrit, une dissymétrie que Louise Dabène (1975, citée par Caddéo dans Cortés Velásquez, 2015, p.x) explique, entre autres, de cette façon: les langues sont plus conservatrices dans leur convention graphique que dans leur système phonologique. Il suffit de penser aux consonnes étymologiques du français dont le corpus lexical s'avère souvent compréhensible par des locuteurs de langues latines à l'écrit, mais pas à l'oral (les substantifs corps, temps, loup, porc, par exemple, sont beaucoup plus transparents pour des romanophones quand ils les voient écrits que lorsqu'ils les entendent, à cause des consonnes finales non prononcées). La capacité à décoder certains termes à l'écrit ne se vérifie donc pas forcément à l'oral, le transfert de la transparence ne se produisant pas automatiquement.

D'autre part, le document écrit n'est guère volatile : il peut être relu plusieurs fois (avec des moments de pause, de réflexion), ce qui facilite son intelligibilité et rend sa lecture moins stressante. Il ne comprend pas non plus d'éléments parasites comme des hésitations, des répétitions, des tics de langage. "L'écrit par rapport à l'oral est rassurant, même lorsqu'on se trouve en présence d'une langue inconnue, à condition bien sûr qu'elle partage au moins les signes graphiques [...]. Le message oral se construit dans l'immédiateté et se caractérise par son évanescence. » (Caddeo \& Jamet, 2013, p. 87) S'ajoutent des habitudes perceptives de la langue maternelle qui constituent un frein, une sorte de filtre altérant la perception sonore d'une langue étrangère ayant une autre musicalité. Autant de raisons - évoquées ici très brièvement - qui expliquent pourquoi l'écrit a souvent été privilégié jusqu'ici en IC.

\section{Une carence de matériel didactique consacré à l'interproduction ${ }^{2}$ orale en IC}

5 Si l'oral est bien présent dans un manuel d'intercompréhension comme Eurom5, sur des sites comme Lingalog, Itinéraires romans de l'Union latine ${ }^{3}$ ou le feu Fontdelcat (consacré au catalan) et si certaines parties de la plateforme Galanet prenaient en compte cette dimension, pour ne citer que des ressources bien connues, on remarquera que l'oral est rarement exploité seul pour lui-même mais qu'il est, au contraire, souvent pris 
en compte comme support complémentaire, comme élément qui sert d'appui à l'écrit. Bien souvent, il ne s'agit pas tant de travailler sur l'oral que d'exploiter de l'écrit oralisé. Intrinsèquement lié au texte écrit, soit parce qu'il s'agit d'une lecture à haute voix enregistrée d'un article (comme dans Eurom5), soit parce que la retranscription d'une vidéo est proposée en tant que support aux dialogues (comme dans Itinéraires romans), l'oral fait un peu office de parent pauvre de l'écrit, même si en IC les deux aspects vont de pair, une modalité servant d'auxiliaire à l'autre réciproquement. En effet, l'écoute d'un texte permet de se familiariser avec la prononciation des mots et peut contribuer à une meilleure compréhension; à l'inverse, visualiser la transcription d'un vocable opaque entendu peut éclaircir un doute. Par ailleurs, dans l'espace d'autoformation de Galanet, des situations intercompréhensives orales sont présentées : il ne s'agit toutefois pas d'interactions plurilingues entre participants, mais bien de modules qui " cherchent, avant tout, à susciter chez les apprenants une réflexion, de dimension à la fois métalinguistique et pragmatique, sur des interactions plurilingues, qui pourra être réinvestie dans une situation authentique » (Baqué et coll., 2007).

6 En outre, dans le matériel didactique actuel consacré à l'IC, apparaissent presque exclusivement des activités visant à améliorer les compétences en compréhension orale. Celles mettant l'accent sur la production orale sont presque absentes, conformément au projet initial de l'intercompréhension (améliorer les compétences de réception), mais les choses ont évolué et ce manque se doit d'être comblé. Si l'on ne peut nier que l'interaction est davantage prise en compte aujourd'hui, notamment l'interproduction (comme en témoignent les descripteurs et indicateurs disponibles sur EVAL-IC sur lesquels nous nous attarderons plus loin), il y aurait lieu de combler cette carence de matériel pour préparer les apprenantes à intervenir dans le cadre d'un dialogue intercompréhensif. Pour qu'il y ait réception il faut qu'il y ait production, et quand le message oral émis ne provient pas d'un écran ou d'un haut-parleur, mais d'une personne en chair et en os cherchant à transmettre une information, la situation est plus anxiogène pour le/la destinataire.

7 Le seul site qui, à notre connaissance, proposait de vraies activités centrées sur l'interaction à l'oral était Eu\&I (Eu-Itercompréhension). Il n'est malheureusement plus actif actuellement, comme d'autres sites d'intercompréhension qui, faute de financements probablement, ont disparu ou n'ont plus du tout évolué une fois le projet terminé. Une réelle perte car ce site proposait des jeux interactifs bien pensés et des vidéos extrêmement précieuses qui, non seulement fournissaient des clés aux apprenants pour apprendre à détecter le sens d'un message en tenant compte du contexte, mais encore et surtout mettaient en scène des récits racontés par des personnes recourant à des techniques intercompréhensives afin de se faire comprendre (gestes, mimiques, intonation...).

8 Il existe également deux courtes vidéos ${ }^{4}$ fort bien conçues mettant en scène un touriste francophone en visite à Barcelone et un autochtone, même si elles ne sont pas réellement didactisées. On y voit comment ces deux hommes parviennent à interagir en parlant chacun sa langue. L'accent est mis sur les procédés qu'ils ont pu trouver pour arriver à résoudre quelques incompréhensions et à communiquer de manière satisfaisante. En fin de compte, le bilan des ressources disponibles est donc bien maigre. Lorsqu'il s'agit de repérer des exemples de mises en situation de personnes pratiquant l'intercompréhension dans la vie quotidienne, de dialogues dans deux ou trois langues différentes, force est de constater qu'on se retrouve bien démunis. De plus, ce matériel 
est soit de piètre qualité (conçus par des non professionnels ni enseignants ni experts en IC), soit peu exploitable en classe ou encore peu représentatif d'un échange dialogique intercompréhensif. Sur YouTube, Linguamix Unistra met à disposition des situations humoristiques de dialogues entre deux locuteurs de nationalités différentes où abondent les malentendus destinés à faire rire. Ce n'est pas l'intercompréhension qui est mise en relief, mais l'incompréhension (!) entre les deux personnes jouant la scène. Des malentendus se produisent, comme si l'objectif était de créer une situation comique, ce qui suscite l'effet inverse de celui qui est recherché car il jette un discrédit sur l'IC.

9 Citons néanmoins une initiative individuelle apparue assez récemment sur la Toile: celle d'un jeune linguiste polonais ayant créé un site $^{5}$ complété par des vidéos ludiques intercompréhensives. L'idée est de mettre en relation des personnes de nationalités diverses issues du monde latin et de demander à l'une d'elles de faire deviner cinq vocables de sa langue aux autres participants. S'ensuit une comparaison entre la traduction du mot à deviner dans différentes langues romanes (y compris régionales). Un jeu sympathique qui permet aux spectateurs de ces vidéos d'apprendre à se faire comprendre par des étrangers et d'écouter des interactions plurilingues.

\section{Le degré de transparence entre langues romanes}

Si l'on peut affirmer sans hésitation que, dans le cas de langues voisines, l'intercompréhension est plus simple qu'entre langues appartenant à des familles de langues différentes, c'est-à-dire que l'intercompréhension intrafamiliale est plus aisée que l'intercompréhension interfamiliale (et ceci vaut tant pour l'oral que pour l'écrit), il convient cependant d'apporter quelques précisions.

11 Certaines langues latines sont plus compréhensibles que d'autres pour les autres locuteurs romans, pour différentes raisons. Par exemple le fait qu'une langue possède des phonèmes qui n'existent pas dans une autre nuit à l'intelligibilité. L'exemple classique est le cas des voyelles nasales du français et, dans une moindre mesure, du portugais, qui constituent indéniablement un écueil pour les locuteurs des autres langues latines. D'autres paramètres interviennent comme l'accentuation, la segmentation, le nombre de phonèmes présents dans une langue ou encore sa propre histoire. Si l'on prend le cas du roumain, les emprunts aux langues slaves sont légion, ce qui fait inévitablement écran. De plus, son système verbal diffère des autres langues romanes et certains aspects morphosyntaxiques peuvent déconcerter d'autres romanophones (Garbarino, 2009, p. 4-5).

12 En travaillant parallèlement sur l'oral et l'écrit, comme c'est souvent le cas en IC, le texte permettra d'associer la prononciation des mots à leur transcription graphique, de "visualiser » les sons entendus (ceci vaut particulièrement pour les langues ayant un degré de transparence orthographique faible comme le français). L'inverse est également vrai : l'écoute d'un terme dont la graphie déroute peut permettre de mieux percevoir ce qui apparait comme illisible et incompréhensible sur papier.

13 Le lexique permet l'entrée dans une langue, il est

[...] la première ressource véritablement linguistique à laquelle l'apprenant fait appel, les autres étant surtout de nature pragmatique, gestuelle, prosodique ou relationnelle. En effet, tout en ayant une connaissance très faible de la morphologie on pourra comprendre approximativement de quoi parle notre interlocuteur si 
nous comprenons (ou devinons) le sens du radical des mots qu'il utilise, si nous

repérons leur champ lexical et sémantique. (REFIC, 2015, p. 24)

Les données fournies par l'Ethnologue, synthétisées dans le tableau 1 ci-dessous, indiquent le pourcentage de ressemblance lexicale entre les six principales langues romanes. Si certaines langues possèdent un haut taux de similitude lexicale entre elles (89\% entre le français et l'italien, idem entre l'espagnol et le portugais), d'autres combinaisons de langues présentent un pourcentage un peu moins élevé : le roumain est assez éloigné du groupe ibérique - tout comme le français d'ailleurs, qui est plus proche de l'italien et du catalan. Dans tous les cas, on voit clairement qu'un fond lexical commun est bien présent et que cette ressemblance se doit d'être exploitée davantage au niveau pédagogique.

Tableau 1. - Pourcentage de ressemblance lexicale entre les six principales langues romanes.

\begin{tabular}{|l|l|l|l|l|l|l|}
\hline$\%$ & Portugais & Espagnol & Catalan & Français & Italien & Roumain \\
\hline Portugais & & 89 & 85 & 75 & 80 & 72 \\
\hline Espagnol & 89 & & 85 & 75 & 82 & 71 \\
\hline Catalan & 85 & 85 & & 85 & 87 & 73 \\
\hline Français & 75 & 75 & 85 & & 89 & 75 \\
\hline Italien & 80 & 82 & 87 & 89 & & 77 \\
\hline Roumain & 72 & 71 & 73 & 75 & 77 & \\
\hline
\end{tabular}

Tableau conçu à partir des données de Ethnologue, Languages of the World, $15^{\mathrm{e}}$ édition, SIL International, 2005.

\section{Le principe de coopération}

Le principe de coopération (Grice, 1979), à l'œuvre dans toutes les conversations, est encore plus fondamental dans un échange oral en intercompréhension, car l'immédiateté de celui-ci ne permet pas à celui ou celle qui écoute de réfléchir aux propos prononcés. Escudé préfère invoquer la notion d'intentionnalité ${ }^{6}$ (Escudé \& Janin, 2010, p. 40 et Escudé, 2016, p. II), c'est-à-dire que l'intercompréhension fonctionne s'il y a une réelle volonté de communiquer chez les interlocuteurs, ce qui les oblige à s'imposer une exigence de clarté et de simplicité, à mettre en place certaines techniques (voir point 5) tout en ayant la perception d'un « sentiment d'appartenance commune » et en exploitant les similitudes. "A comunicação é um ato volitivo: depende da vontade e da predisposição dos interlocutores. " (Escudé \& Calvo del Olmo, 2019, p. 58) Aucun prérequis n'est réellement exigé - même si être professeure de langue ou linguiste peut aider -, mais un réel désir de comprendre l'Autre dans sa diversité linguistique et de vouloir se faire comprendre est un bon point de départ. C'est en cela que l'IC est une approche altruiste, éthique et équitable. Le souhait d'aller à la rencontre d'une personne ne parlant pas la même langue prime; "la réussite de l'échange repose essentiellement sur la bonne volonté des participants qui assurent 
conjointement le pilotage de l'interaction en coordonnant et en harmonisant en permanence leurs comportements respectifs. Lorsque tous les participants tiennent convenablement leur rôle en l'ajustant à ceux tenus par les autres, l'interaction est considérée comme réussie » (Louis, 2007, p. 189). C'est ce principe même de négociation qui fait de cette modalité communicative une approche humaniste, basée sur le respect de l'autre, l'intérêt réciproque, la parité. Faire un effort pour comprendre la langue de l'autre témoigne d'une attitude d'ouverture. L'inverse est vrai également: chercher à être intelligible en adoptant certains «trucs» est une façon de ménager son interlocuteur.

\section{Stratégies verbales et non verbales}

15 Si en linguistique l'analyse des interactions verbales n'est plus récente, on peut s'étonner que cet aspect soit souvent sous-estimé aujourd'hui en didactique des langues, notamment en ce qui concerne la multimodalité ${ }^{7}$ de la communication, peu enseignées depuis l'avènement de l'approche communicative ${ }^{8}$. Étant donné qu'elle fait partie intégrante des échanges conversationnels, il nous semble important que, à un moment ou un autre du parcours didactique, les enseignantes de langue sensibilisent leurs élèves aux aspects non verbaux présents dans les parties dialogiques des documents vidéos qu'ils projettent en salle de cours. Lors d'un exercice de compréhension orale (vidéo), les mimiques des personnages, leur expression faciale et corporelle, pourraient davantage faire l'objet d'une analyse. Une leçon entièrement consacrée à la gestualité utilisée par les locuteurs/trices de la langue cible voire à leur kinésie s'avèrerait également utile, car l'on sait que les gestes ne sont pas identiques dans toutes les cultures et peuvent être source de malentendus. Dans les interactions exolingues ${ }^{9}$, qu'elle soient unilingues ou plurilingues, et comme Oursel le souligne "il est nécessaire que l'apprenant-interactant, en tant que locuteur, apprenne à adapter sa production à son interlocuteur pour lui faciliter la tâche de l'interprétation, et en tant qu'interlocuteur, apprenne à gérer la construction de ses hypothèses d'interprétation " $(2019, \S 35)$. Il en va de même lorsque deux personnes interagissent chacune en parlant une langue différente. Dans cette optique, nous aimerions à présent dresser un inventaire (non exhaustif) des stratégies qu'il est opportun de mettre en pratique à l'oral lorsque l'on discute de façon intercompréhensive. Des «techniques » qui ne sont pas propres aux échanges plurilingues (les enseignantes de langues étrangères les appliquent de manière plus ou moins consciente), mais qui sont d'autant plus recommandées que les interlocuteurs s'expriment dans des langues différentes pour communiquer. Cette liste devrait permettre aux formateurs et formatrices de pouvoir fournir des conseils pratiques aux apprenantes dans l'objectif de communiquer de façon efficace. Elle devrait idéalement préluder à des jeux de rôles.

\subsection{Techniques verbales}

16 Il est recommandé de/d' :

1) utiliser un lexique « transparent " (internationalismes et lexique panroman)

17 Il s'agit tout d'abord d'utiliser des internationalismes. En effet, certains vocables, sont soit identiques soit très semblables dans de nombreuses langues. Si l'on utilise taxi, radio, Internet, hôtel, alcool, on sait que l'on sera compris presque aux quatre coins de la 
planète. Il s'agit également d'anticiper les mots opaques afin de faciliter l'échange, de prévoir ce qui pourrait causer problème. Cette stratégie quelque peu intuitive repose sur la conscience du risque d'opacité, donc de la capacité à se décentrer. Il est évident qu'avoir un peu de sensibilité linguistique et de pratique aide: avec le temps, en procédant par tentatives, l'apprenant·e saura que certains termes posent des problèmes de compréhension alors que d'autres sont plus aisément identifiables. Grâce à une formation en IC, il/elle apprendra à faire usage de mots congénères ${ }^{10}$ (p.ex. les adverbes en "ment" existent dans toutes les langues latines, sauf en roumain, ce qui explique pourquoi « rapidement» est préférable à "vite»). Un francophone comprendra que "pareil » en français est plus opaque que «similaire » pour d'autres locuteurs de langues latines (rapprochable, de surcroit, avec l'anglais «similar»). De même, un italophone parlera de "rapidità » plutôt que de "velocità " avec un francophone. Les mots d'origine savante et le lexique technique, souvent basés sur une racine gréco-latine (REFIC, 2015, p.67) sont également à favoriser, car la langue de spécialité est souvent partagée par toutes les langues romanes (et pas uniquement).

2) reformuler, trouver des synonymes, dire de manière différente (paraphrase)

En IC, entre deux interlocuteurs se crée un genre de "pacte » implicite : il s'agit de se mettre d'accord tacitement, en quelque sorte, sur des termes que l'on comprend. Lorsqu'un terme n'est pas compris, il faut trouver un synonyme compréhensible. Une fois trouvé, il devra continuer à être employé. Les termes les plus courants de la vie quotidienne sont souvent les plus opaques. À l'inverse, certains mots désuets ou littéraires sont parfois plus aisés à comprendre. Reformuler s'avère essentiel: expliquer, définir, fournir un hyperonyme voire une traduction (point suivant), c'est donner une deuxième ou troisième chance à la personne avec qui on communique, c'est veiller à ne pas l'isoler en se donnant des moyens conversationnels supplémentaires d'accéder au référent.

3) recourir à une langue tierce ponctuellement

19 Dans une démarche intercompréhensive, utiliser un terme appartenant à une langue tierce affine est la première démarche à entreprendre, mais rien n'empêche d'utiliser un mot anglais ou un mot d'une autre langue au besoin, de manière ponctuelle.

4) éviter les noms propres, faire une périphrase

Les noms propres sont traduits dans certaines langues et demeurent opaques pour des étrangers. Qu'il suffise de penser à Topolino ou Paperino en italien (respectivement Mickey Mouse et Donald Duck) : mieux vaut les nommer dans leur langue originale. Ainsi, on pourrait dire la reine d'Angleterre, la capitale du Portugal, la petite fille vêtue en rouge qui rencontre un loup dans la forêt...

5) utiliser une langue standard (registre "neutre", mais naturel) et limiter la longueur des énoncés

21 Il importe d'émettre des énoncés simples et brefs, car la longueur excessive d'un énoncé constitue un obstacle. Par ailleurs, l'argot, le langage branché, le jargon, les termes familiers sont à éviter, car ils risquent de ne pas être compris. "Cigarette » est facilement compréhensible par des étrangers, y compris non romanophones, alors que «clope » ne l'est pas. Par contre, il arrive qu'un terme peu usité (de registre soutenu) soit plus intelligible que le terme standard. Le terme "espelma» en catalan est incompréhensible pour d'autres romanophones, mais « candela ", peut être compris par tout le monde et «bugia », moins fréquent, est transparent pour les francophones. 


\section{6) répéter un même mot}

Répéter un mot non compris est évidemment une chose que l'on fait naturellement. Cependant, lorsqu'un mot est opaque, ce procédé est inutile. Après deux ou trois tentatives, il est nécessaire de changer de stratégie. Pour permettre le maintien du référent, la reprise lexicale pleine est préférable à l'usage d'un pronom : elle permet à l'auditeur de mieux traiter la donnée langagière.

7) utiliser des onomatopées

23 Cette technique peut faire sourire, mais dans certains cas pourquoi ne pas y recourir? S'il est vrai que les onomatopées diffèrent d'une langue à l'autre, beaucoup se ressemblent ou sont carrément identiques. Une onomatopée vaut parfois mieux qu'un long discours et ajoute une touche d'humour à la conversation.

8) donner des exemples

Ce dernier élément est en lien direct avec les aspects linguistiques, tout en étant une stratégie pragmatique. Illustrer une pensée, un concept par des exemples permet de concrétiser ses propos, de clarifier une notion, de passer d'une idée abstraite à un cas concret.

9) éviter les sigles / acronymes, les abréviations, les troncations

Les Français sont les champions en la matière. Cela peut sembler évident, mais il est bon de rappeler aux étudiants francophones que parler de SDF, de TVA, de SIDA, de pharma, de géo, de perfu ou de dirlo nuit grandement à la compréhension, car les sigles et les troncations, en général, demeurent obscurs pour des non-francophones voire pour des non-initiés de la même communauté linguistique. Il convient donc de dire les (groupes de) mots de façon complète.

10) éviter les idiomatismes / expressions figées

Tout ce qui relève des expressions idiomatiques ou parémiques (locutions figées, formules routinières, proverbes) est à éviter. Bien que de nombreuses tournures idiomatiques soient identiques ou semblables dans différentes langues, leur caractère imagé risque de les rendre non compréhensibles. Il est donc déconseillé d'insérer des expressions à prendre au sens figuré dans une conversation.

\section{1) S'appuyer le plus possible sur un univers culturel partagé}

Certains vocables sont très connotés culturellement et n'ont pas d'équivalent dans d'autres langues, car ils sont liés aux connaissances sociales et culturelles partagées par une communauté (mœurs, traditions, etc.). Par conséquent, il est recommandé de modérer l'usage de notions à forte « charge » culturelle et de les expliciter lorsqu'on les utilise. Le mieux est d'essayer de rester dans un monde culturel universel, accessible à tous, en ayant conscience que certains blocages à la compréhension peuvent provenir de divergences dans les valeurs, pratiques et références des interlocuteurs. Ceci signifie également manier avec précaution l'ironie et l'humour qui pourraient être mal interprétés.

Toutes les stratégies verbales que nous venons de citer, de manière non exhaustive, sont susceptibles de devenir des critères d'évaluation des compétences en interproduction et interaction. Comment deux étudiant(e)s sont-ils parvenus à communiquer? Quel éventail de stratégies ont-ils déployé pour se faire comprendre? Comment ont-ils remédié aux difficultés communicatives, aux malentendus, aux ruptures conversationnelles? Bref, ont-ils communiqué de façon efficace? 
29 Dans le projet EVAL-IC ${ }^{11}$, trois critères sont envisagés par les descripteurs (et indicateurs) relatifs à l'interproduction orale pour la dimension linguistique ${ }^{12}$ : l'étendue, l'adéquation et la disponibilité. Le premier critère consiste à inclure volontairement des éléments linguistiques discursifs facilitant la compréhension : il s'agit donc d'adapter son discours afin de faciliter la compréhension pour l'interlocuteur/trice, ce qui signifie choisir un certain type de lexique, recourir à des formes redondantes (utilisation de synonymes, d'hyperonymes, etc.) et opter pour une syntaxe adaptée. Le lexique est surtout pris en considération par le paramètre de l'adéquation, c'est-à-dire la capacité à adapter son répertoire lexical en fonction de l'interlocuteur/trice. La capacité à éviter les mots argotiques, le langage familier, les abréviations ou les sigles intervient dans ce paramètre, mais aussi le choix d'utiliser des mots que l'on suppose assez transparents. Il s'agit (au départ), en effet, de suppositions, de tentatives : on procède à tâtons en quelque sorte. Le critère de disponibilité va dans le même sens. Il s'agit ici de se montrer disposé à répéter, à reformuler, à faire une périphrase, bref, à être en mesure de « dire d'une autre manière ».

EVAL-IC propose différents niveaux de compétence allant de 1 à 6 . Au premier niveau, le/la locuteur/trice n'utilise que quelques moyens de base (quelques mots internationaux, des éléments non verbaux limités) et n'assure pas réellement la compréhension de ses interlocuteurs alors qu'une personne de niveau 6 est en mesure de sélectionner «les stratégies (verbales, paraverbales et non verbales) les plus adéquates pour garantir la pleine compréhension de son discours, tout en maintenant un niveau de complexité discursive important ${ }^{13}$ ». Cela signifie que la personne est tout à fait apte à recourir à des stratégies comme la reformulation, l'usage de mots non opaques et qu'au fur et à mesure, elle parviendra à éviter la redondance lexicale en optant directement pour le terme le plus transparent.

\subsection{Techniques paraverbales / non verbales}

31 Outre les aspects strictement verbaux présents dans un dialogue interviennent d'autres éléments qui sont généralement peu abordés en classe, comme si leur didactisation était considérée comme superflue, comme si les cours de langue ne devaient se focaliser que sur les aspects purement linguistiques d'une conversation. Le contexte, pour commencer, aide énormément à décrypter le sens d'un échange conversationnel. Il est fort peu probable, en effet, que deux personnes qui se trouvent dans une pharmacie de part et d'autre du comptoir discutent de musique ou de zoologie. À priori, les participants peuvent imaginer qu'on a affaire à un dialogue entre un client et une pharmacienne à propos d'un médicament, par exemple. La situation fournit donc des indices sur le contenu de l'échange et l'endroit où il se déroule est connoté socialement. "Chaque lieu (que ce soit le palais de justice, le bureau de poste ou le cabinet du médecin, etc.) induit une sorte de scénario culturel ou de guide de bonne conduite particulier qui fait l'objet d'un consensus auprès des membres de la communauté. » (Kerbrat-Orecchioni, 1990, p. 78). Ceci est valable également, du moins en partie, pour des interactions entre des personnes appartenant à deux univers culturels différents, car certaines situations sont universelles (demander une information, commander une boisson, déclarer son identité...).

32 Par ailleurs, les éléments paraverbaux et non verbaux sont également porteurs de sens et méritent non seulement d'être étudiés, mais aussi, lorsque l'on veut former des 
apprenant·es à l'interproduction orale, à être enseignés. Nous insérons ici également des stratégies relevant plutôt de la compétence pragmatique. Ainsi, dans un dialogue, est-il recommandé de/d' :

1) exprimer des sentiments par le visage, par des mimiques

En intercompréhension, tout comme dans n'importe quelle situation où une personne est confrontée à une langue étrangère, qu'elle la parle ou non, l'expression du visage joue un rôle plus important que dans une conversation entre deux personnes parlant le même idiome. Le visage devient un paysage à observer, une sorte de tableau à interpréter. D'où l'importance d'accentuer quelque peu les mimiques faciales.

Le visage transmet des informations de plusieurs natures. Celles-ci sont d'abord de nature émotionnelle. [...] S'y ajoutent des éléments de nature kinésique, que l'on accompagne une interrogation d'un mouvement de tête ou que l'on souligne par une mimique un accord, une liaison qui apporte un complément d'information. Par ailleurs, des éléments d'ordre articulatoire de nature plus strictement linguistique - le resserrement des lèvres, leur arrondissement, l'écartement des maxillaires - sont des informations complémentaires utiles pour la compréhension langagière. Ce sont en général des éléments plus pertinents en situation d'apprentissage d'une langue étrangère, mais tout aussi utiles pour la perception de tous types de messages. (Abou Haidar, 2020).

2) parler lentement en articulant bien

34 Tout ce qui relève du paraverbal (débit, pauses dans la diction, intensité articulatoire...) revêt une grande importance dans un échange intercompréhensif et, de façon générale, lorsqu'il s'agit de comprendre une langue étrangère. Si modérer son débit est primordial pour se faire comprendre, on oublie souvent que l'économie articulatoire peut freiner la compréhension, d'où l'importance de veiller à la prononciation. Le défi consistera à segmenter le flux de paroles sans y perdre en expressivité, en essayant de ne pas se laisser emporter par le contenu de la discussion.

3) regarder son interlocuteur/trice quand on parle

Inutile de préciser que le regard est un élément à ne pas sous-estimer. Il permet de soutenir l'attention, d'accorder une certaine importance à son interlocuteur (hormis certaines situations où baisser le regard peut être une marque de respect). «C'est par le visage que passent des informations de nature prosodique ou "musicale" d'ordre macrokinésique, très étroitement liés à la gestuelle globale du corps, et à la microkinésie vocale.» (Abou Haidar, 2020) Voilà pourquoi le regard s'avère essentiel. Le visage délivre des informations et la perception visuelle de celles-ci facilite leur mémorisation.

4) s'assurer que l'interlocuteur/trice a compris, qu'il/elle suit le fil

Fournir un feedback et attendre un retour de l'autre permet de remédier aux ratés de la conversation. Faire semblant de comprendre est parfois une stratégie presque obligée pour ne pas perdre la face ou la faire perdre à son interlocuteur/trice. Cependant, afin d'éviter un quiproquo en cascade, il est préférable de demander un éclaircissement ou encore reformuler dans sa langue ce que l'on croit avoir compris. Cela permet aux deux interlocuteurs de s'assurer réciproquement du bon fonctionnement de l'échange, "mais aussi de consolider la compétence plurilingue des interlocuteurs grâce à la reformulation du même contenu dans une autre langue » (REFIC, 2015, p. 68).

5) mettre l'accent sur les termes importants (intonation) 
Il s'agit de mettre en relief certains termes afin d'en souligner l'importance par une légère insistance intonative. Dans un flux verbal, certains mots sont superflus ou, du moins, sont moins primordiaux que d'autres. Il importe de marquer une distinction dans la diction en accentuant les contenus informationnels prioritaires (et non pas de hausser la voix, comme certaines personnes font, pensant aider leur interlocuteur/ trice!).

6) faire des gestes si c'est nécessaire sans en abuser

38 C'est un point central parmi les stratégies. Qu'ils soient déictiques (indiquer un référent, plus ou moins abstrait), des «emblèmes» (gestes conventionnels qui remplacent le langage parlé), iconiques (dont la forme fait référence à des traits emblématiques de l'objet), voire métaphoriques (qui portent sur la grammaticalité du discours et dont le référent est abstrait), pour reprendre les catégories de McNeill (McNeill, 1992, cité dans Gastal, 2018, p. 23-25), les gestes revêtent toute leur importance. Ils pallient les carences langagières, complètent le discours, le renforcent, servent d'auxiliaires lorsque l'incompréhension prend le dessus. Les gestes sont véritablement une duplication de la parole bien que, là aussi, ils varient d'une culture à l'autre.

7) s'aider d'objets présents autour de soi ou de supports visuels

39 Cette stratégie est moins prégnante que les précédentes, car il n'est pas toujours possible d'avoir des objets à disposition. Elle peut néanmoins s'avérer utile dans certains cas. Par ailleurs, durant un exposé, d'une narration (raconter une histoire, un conte), fournir un support visuel peut grandement faciliter la compréhension de la part du public.

8) faire un dessin, un schéma, un plan

Comme pour le point précédent, ce «truc » n'est pas toujours réalisable sur un plan pratique. Cependant, s'il y a rupture dans la communication et que la situation le permet, pourquoi ne pas recourir au dessin ? Il est parfois plus simple d'écrire le prix à payer dans un restaurant ou hôtel avec le détail que de l'expliquer.

41 Pour tous ces aspects non verbaux et paraverbaux, nous retrouvons dans EVAL-IC les trois critères cités précédemment (étendue, adéquation, disponibilité). Le premier critère se réfère à la capacité à segmenter son propre flux de paroles de manière à ce que la personne que l'on a en face de soi parvienne à repérer les termes séparément malgré les liaisons, élisions, etc. En effet, il est fréquent de voir certaines personnes oublier complètement après quelques répliques qu'elles dialoguent avec un·e locuteur/ trice non natif/ve et débiter un flux de paroles sans tenir compte des problèmes de compréhension que peut créer leur discours. Leur effort n'est pas maintenu dans la durée. À l'inverse, une personne attentive veillera constamment à segmenter ses phrases, cherchera à être claire quitte à, de temps à autre, atténuer son expressivité. EVAL-IC met d'ailleurs l'accent sur la régulation du débit et l'importance de ne pas mâcher ses mots, c'est-à-dire de bien articuler. Quand on ne connait pas les besoins de son interlocuteur, on les imagine, ce qui signifie qu'une part d'intuition entre en jeu. À ces qualités s'ajoutent l'importance de la posture adoptée, les mouvements, l'expression du visage: autant d'éléments non verbaux qui devraient, dans une démarche intercompréhensive, faciliter l'échange (qu'il suffise aux multiples manières d'exprimer une négation, un désaccord), l'idéal étant de recourir à ces éléments 
" en concomitance avec le verbal pour transmettre des informations additionnelles " en tenant compte du fait qu'il ne s'agit pas d'une communication monolingue.

\section{Conclusion}

Nous avons voulu contribuer à inciter les formatrices et formateurs en intercompréhension à travailler davantage l'interproduction orale en classe, c'est-àdire à inciter les étudiantes à adapter leur production en langue maternelle (ou autre langue bien maitrisée éventuellement), à mettre en place des stratégies pour mieux communiquer, tout en les mettant en relation avec les critères / paramètres établis par EVAL-IC, qui s'avère être un outil extrêmement utile pour les formateurs/trices et évaluateurs/trices. Grâce à des conseils simples et concrets mais indispensables, il est envisageable d'enseigner et d'exercer les principes de l'intercompréhension et même d'évaluer leur mise en application grâce à EVAL-IC. Des jeux de rôles - qui mettront en scène de préférence des situations réelles (tourisme, affaires, etc.) devraient idéalement être proposés (ceci est rendu possible dans une classe plurilingue) à condition qu'ils soient précédés d'une séance au cours de laquelle l'enseignant·e veillera à fournir aux apprenantes les instruments qui leur permettront de communiquer oralement de façon la plus efficace possible par le visionnement de vidéos par exemple. Un tel parcours didactique, focalisé sur l'interproduction orale, devrait pouvoir pallier un manque en accordant sa juste valeur à un aspect jusqu'à présent trop longtemps laissé de côté et se rapprocher davantage du sens originel de l'intercompréhension. Il devrait également et idéalement servir à mieux communiquer de façon générale de manière naturelle dans tout type de situation.

\section{BIBLIOGRAPHIE}

ABOU HAIDAR, Laura. (2020, 6 mai). Enseigner avec un visage masqué : un défi ? The Conversation France. <www.theconversation.com>.

BAQUÉ, Lorraine, LE BESNERAIS, Martine \& MARTIN, Éric. (2007), Le traitement de la compréhension orale sur Galanet, dans F. Capucho, A. Martins, C. Degache \& M. Tost, Diálogos em Intercomprensão (p. 375-408). Universidade Católica Editora.

CADDÉO, Sandrine \& JAMET, Marie-Christine. (2013). L'intercompréhension : une autre approche pour l'enseignement des langues. Hachette FLE.

CAPUCHO, Filomena. (2017, juillet). Interactions professionnelles plurilingues : entre intercompréhension et interproduction. Les z'oraux - Les français parlés entre sons et discours, Repères DoRiF, 12. <www.dorif.it/reperes/filomena-capucho-interactions-professionnellesplurilingues-entre-intercomprehension-et-interproduction/>.

CORTÉS VELÁSQUEZ, Diego. (2015). Intercomprensione orale. Ricerca e pratiche didattiche. Le Lettere. 
DE CARLO, Maddalena \& ANQUETIL, Mathilde. (2019). Un Référentiel de compétences de communication plurilingue en intercompréhension (REFIC). EL.LE, 8(1), 163-234. <https:// edizionicafoscari.unive.it/media/pdf/article/elle/2019/1/art-10.14277-

ELLE-2280-6792-2019-01-008.pdf ${ }^{>}$.

EsCuDÉ, Pierre (dir.). (2016). Autour des travaux de Jules Ronjat, 1913-2013. Unité et diversité des langues. Théorie et pratique de l'acquisition bilingue et de l'intercompréhension : actes de colloque. Éditions des archives contemporaines.

ESCUDÉ, Pierre \& CALVO DEL OLMO, Francisco. (2019). Intercomprensão. A chave para as linguas. Parábola editorial.

ESCUDÉ, Pierre \& JANIN, Pierre. (2010). Le point sur l'intercompréhension, clé du plurilinguisme. CLE International.

EVAL-IC (Évaluation des compétences en intercompréhension), Projet de recherche. <https://evalic.eu>.

FERRE, Gaelle. (2011). Analyse multimodale de la parole. Rééducation orthophonique, 246, 73-85.

<https://hal.archives-ouvertes.fr/hal-00609124/document>.

GARBARINO, Sandra. (2009). Le roumain est-il vraiment une langue voisine ? Dans M. H. Araújo é

Sá, R. Hidalgo Downing, S. Melo-Pfeiffer, A. Séré \& C. Vela Delfa (dir.), A Intercompreensão em Línguas Românicas (p. 197-212). Oficina Digital.

GASTAL, Aurore. (2018). Analyse des gestes spontanés à visée communicative chez quatre adolescents paralysés cérébraux dont l'intelligibilité est réduite en dépit de troubles moteurs prédominants : quand l'intention dépasse l'imperfection de la réalisation (Mémoire de recherche). Université de Nice Sophia Antipolis, Faculté de médecine.

GRICE, H. Paul. (1979). Logique et conversation. Communications, 30, 57-72. <https://doi.org/ 10.3406/comm.1979.1446>.

JAMET, Marie-Christine (dir.). (2009). Orale e intercomprensione tra lingue romanze. Ricerche e implicazioni didattiche. Le Bricole.

JAMET, Marie-Christine. (2010). L'intercompréhension : de la définition d'un concept à la délimitation d'un champ de recherche ou vice versa.Publif@rum, Autour de la définition, 11, 100-110. <www.publifarum.farum.it/index.php/publifarum/article/view/193/1056>.

KERBRAT-ORECCHIONI, Catherine. (1990). Les interactions verbales (t. 1). Armand Colin.

LoUIS, Vincent. (2007). Interactions verbales et communication interculturelle en FLE. De la civilisation française à la compétence (inter)culturelle. E.M.E.

OURSEL, Élodie. (2019). Apprendre à gérer l'intercompréhension en langue étrangère. Analyse du processus, des stratégies et des paramètres dans une perspective didactique. Linx, 79. <https:// doi.org/10.4000/linx.3571>.

PORQUIER, Rémy. (1984). Communication exolingue et apprentissage des langues. Dans B. Py (dir.), Acquisition d'une langue étrangère : actes de colloque (p. 17-47). Secrétariat de l'Université de Neuchâtel.

Référentiel de compétences de communication plurilingue en intercompréhension (REFIC). (2015). Projet collectif coordonné par M. De Carlo dans le cadre du programme européen MIRIADI. $<$ www.miriadi.net/referentiels>. 
TombolinI, Annalisa. (2018). Intercompréhension orale entre langues romanes : stratégies cognitivesémotionnelles et implications didactiques à travers une recherche-action (Thèse de doctorat en sciences du langage). Université Ca' Foscari, Venise.

\section{NOTES}

1. Voir les travaux récents de Tombolini (2018) et Cortés Velásquez (2015) par exemple.

2. «Le terme a été proposé pour désigner la production dans une langue que l'on maitrise suffisamment bien, souvent sa langue maternelle, pour s'adresser à un/des partenaires de communication qui n'a/ont pas forcément appris cette langue et s'exprime/nt, lui/eux, dans une autre langue. » (Capucho, 2017) Cette dénomination un peu controversée est discutable, en effet, car, de fait, on ne voit pas bien ce qui la différencie de l'intercompréhension orale, qui devrait théoriquement comporter un effort de communication envers l'autre. Cependant, son acception est plus précise car elle désigne la capacité d'adapter sa propre production en langue maternelle (ou « langue première ») à l'interlocuteur/trice.

3. Les contes d'Itinéraires romans ne sont malheureusement plus accessibles depuis le $1^{\mathrm{er}}$ janvier 2021 suite à la disparition d'Adobe Flash Player.

4. L'une d'entre elles avait été conçue pour un CLOM consacré à l'IC proposé par l'OIF.

5. <www.ecolinguist.com>.

6. Il reprend en fait l'appellation et le concept que Ronjat avait inventés en 1913 dans Le développement du langage observé chez l'enfant bilingue.

7. Voir à ce sujet l'article de Ferre (2011).

8. Il n'en a pas toujours été de la sorte. En effet, à partir des années 1950 et jusqu'à l'arrivée de l'approche communicative, la méthode SGAV (structuro-globale-audiovisuelle), dont les premiers fondements théoriques ont été fournis par Petar Guberina, prend en compte les éléments non verbaux tels que la gestuelle, l'expression des sentiments et des émotions qui passent à travers le visage.

9. Rémy Porquier entend par communication exolingue une communication établie entre des individus ne partageant pas la même langue maternelle, par opposition à la communication endolingue, qui s'établit entre des individus ayant une langue maternelle commune (1984, p. 17). Nous prenons ici ce mot dans l'acception suivante: une interaction où les deux parties en présence ne parlent pas la même langue et communiquent chacune soit dans leur propre langue, soit dans une langue étrangère bien maitrisée. De ce fait, ils adaptent leurs comportements en conséquence.

10. Environ 500 mots d'origine latine selon Tombolini (2018, p. 15).

11. Il s'agit d'un projet de recherche visant à définir les compétences en IC et à proposer des outils d'évaluation. C'est l'aboutissement d'un partenariat entre 14 universités européennes et une trentaine d'institutions partenaires. Il fournit une sorte de cadre destiné aux formatrices et formateurs en IC. Bien que ces travaux n'aient pas (encore) été réunis en un seul volume, EVAL-IC est aujourd'hui une référence pour la définition des niveaux de compétences et pour l'évaluation en IC.

12. Les dimensions interculturelle et pragmatique ne comprennent qu'un seul critère: l'adéquation.

13. EVAL-IC, Descripteurs généraux Interproduction. 


\section{RÉSUMÉS}

Le présent article se propose de se pencher sur un aspect souvent délaissé en intercompréhension (IC) entre langues affines (romanes, dans ce cas-ci) : l'interproduction orale, c'est-à-dire la capacité d'adapter sa langue maternelle (ou une autre langue bien maitrisée) à son interlocuteur ou interlocutrice dans des interactions intercompréhensives. Il souhaite fournir aux formatrices et formateurs en IC des pistes didactiques visant à enseigner à des jeunes ou à des adultes comment mettre en pratique certaines stratégies verbales ou non verbales de manière à pouvoir les faire interagir de façon efficace lors d'une conversation avec des personnes ne parlant pas le même idiome. Grâce à des conseils pratiques, il est possible et même souhaitable de fournir aux étudiant(e)s des procédés simples et très concrets pour interagir oralement, une sorte de «kit de survie » leur permettant de communiquer avec toute personne étrangère, notamment si celle-ci parle une langue proche de la leur.

The purpose of the current article is to examine an aspect that is often overlooked when talking about intercomprehension (IC) between languages of common origin (in this case Romanic languages): oral interproduction, i.e. the capacity for somebody to adapt his mother tongue (or another well-mastered tongue) to his interlocutor in oral interactions. It wishes to provide IC trainers with didactic clues on how to teach youngsters and adults to put into practice certain verbal or non-verbal strategies so as to make them interact efficiently during a conversation with people who do not speak the same idiom. Thanks to practical advices it is possible and advisable to give students simple and very concrete tricks that will enable them to interact orally, giving them a kind of "survival kit" to communicate with any foreign person, especially if this person speaks a sister language.

\section{INDEX}

Mots-clés : intercompréhension orale, interproduction, stratégies verbales et non verbales Keywords : oral intercomprehension, interproduction, verbal and non-verbal strategies

\section{AUTEUR}

\section{HUGUES SHEEREN}

Università di Firenze

hugues.sheeren@unifi.it 